\title{
Doppler ultrasound in intrauterine growth retardation and its indices in correlation with gestational age
}

\begin{abstract}
Background and objective: Intrauterine growth retardation remains the most frequent cause of perinatal morbidity and mortality. Doppler ultrasound has become an indispensable tool in evaluating pregnancies at risk, on the basis of the vascular resistance to blood flow. This study aimed to evaluate non-invasively the fetoplacental and uteroplacental circulations in clinically confirmed high-risk pregnancies, using Doppler parameters of some vessels to apply clinical interventions which could result in reduced prenatal morbidity and mortality and help to decide the type of delivery.

Methods: This cross-sectional study was conducted in Duhok city from June 2014 to January 2015. The study involved 100 pregnant women between 26-42 weeks of gestation with known last menstrual period and a definite clinical high-risk for intrauterine growth retardation. Various Doppler indices were performed including the resistive index, pulsatility indices of both umbilical and middle cerebral arteries, and the resistive index of the uterine artery to predict fetal health, correlated with physical and clinical outcomes, taking into consideration the variability in Doppler measurements of gestational age. Data have been described and then analyzed statistically for their correlation with gestational age.
\end{abstract}

Results: Umbilical artery and fetal middle cerebral artery Doppler indices are correlated inversely with gestational age. Although the uterine artery has a major role in Doppler examination of high-risk pregnancies, there is only a weak correlation between maternal uterine artery Doppler index and gestational age in late pregnancy. Doppler measurement of three arteries was more conclusive and show more sensitivity than one arterial Doppler measurement in the third trimester.

Conclusion: Significant inverse correlations were found between Doppler indices of fetal middle cerebral and umbilical arteries, and gestational age, while the correlation with maternal uterine artery index was not significant with advancing gestational age.

Keywords: Intrauterine growth restriction; Resistive index; Pulsatility index; Middle cerebral artery; Umbilical artery; Uterine artery.

\section{Introduction}

Intrauterine growth retardation (IUGR) complicates $5 \%$ to $10 \%$ of all pregnancies and up to $30 \%$ of multiple pregnancies. In $60 \%$ of these pregnancies, the primary cause is placental insufficiency. Improvement in the identification of the fetus at risk of intrauterine demise may lead to more successful management strategies. ${ }^{1,2}$ Doppler flow velocity waveform analysis of fetal vessels is one

of the main methods for evaluating fetal health before labor; it is considered one of the major tests for prenatal fetal surveillance to determine fetal wellbeing and to prevent perinatal mortality and morbidity, as well as for the timing of delivery. Vascular indices of various fetal vessels were assessed by Doppler ultrasound. The pulsatility index $(\mathrm{PI})$ and the resistive index (RI) are measured on the umbilical, cerebral, and maternal

* Department of Radiology, Maternity Hospital, Duhok, Iraq.

** Department of Surgery, College of Medicine, Duhok University, Duhok, Iraq. 
uterine arteries. Ratios of these indices are based on the comparison of cerebral, umbilical, and uterine vascular resistance, frequently associated and affected by IUGR, in various sensitivities and specificities. ${ }^{1-3}$ Placental insufficiency is the primary cause of intrauterine growth restriction in normally formed fetuses and can be identified using umbilical artery Doppler velocimetry. ${ }^{1,4}$ Umbilical artery Doppler waveforms provide an estimate of downstream placental vascular resistance and placental blood flow., ${ }^{5,6}$ In case of fetal hypoxia, there is vasodilatation of the cerebral artery and consequently decreased $\mathrm{PI}$ and redistribution of fetal blood flow to the vitally important organs. ${ }^{5,7}$ The resistive index (RI) of the uterine artery has a normal range between $0.45-0.58$ after 26 weeks of gestation; decreased end diastolic flow and consequent raised RI above 0.58 (after 26weeks gestation) is considered abnormal. Multi-vessels Doppler parameters' changes precede changes in the biophysical parameters in the fetus with severe IUGR. ${ }^{7,8}$ Doppler indices are calculated using the pulsatility index and resistive index, which are calculated by certain methods. ${ }^{9,10}$ The normal values of Doppler Velocimetry are umbilical artery (Umb.A): P.I. $=0.6-1.4$, R.I. $=0.5-0.7$; middle cerebral artery (MCA): P.I.>1.3, R.I.>0.7; uterine artery (Ut.A):RI = 0.45-0.58. ${ }^{11-13}$ Donald School Textbook of Ultrasound in Obstetrics and Gynecology contains standard nomograms showing the normal value of each arterial index during each week of gestation. ${ }^{6}$ This study aimed to evaluate non-invasively the fetoplacental and uteroplacental circulations in clinically confirmed high-risk pregnancies, using Doppler parameters of some vessels to apply clinical interventions which could result in reduced prenatal morbidity and mortality and help to decide the type of delivery.

\section{Methods}

This cross-sectional study was conducted from June 2014 to January 2015. One hundred pregnant women with known last menstrual period (LMP), definite clinical high-risk pregnancy and normal fetal morphological ultrasound findings, who were at 26-42 weeks of gestation were selected from the Consultancy Clinic of the Obstetrics Hospital at Duhok. The study protocol was approved by the Scientific and Ethics Committee of the Faculty of Medical Sciences of the University of Duhok. A pretested questionnaire was designed to obtain information about gestational age, last menstrual period, expected date of delivery, presenting complaint, previous obstetrical, medical and surgical histories. The patients were examined using Siemens (ACUSON X3000) machine with $3.5 \mathrm{MHz}$ convex transducer. Grey scale ultrasound was performed first, measuring the fetal biparietal diameter, femoral length, abdominal circumference, estimated fetal weight, placental location and grade of maturation, liquor amount and consistency. Cases with the well-documented length of pregnancy (known LMP), gestational age at 26-42 weeks, high-risk pregnancy as pre-eclampsia, gestational diabetes, twin pregnancy, polyhydramnios and oligohydramnios all were included in the study, while wrong dating, gestational age more than 42 weeks and intrauterine death were excluded from the study.

Statistical analysis: Data management and statistical analyses were performed using the statistical package for social sciences (SPSS) version 22. Data for the 100 women were described and then analyzed with appropriate statistical tests. The variables of patient age, gestational age, $\mathrm{RI}$, and $\mathrm{PI}$ were summarized by their range, mean and standard deviation (SD). The sensitivity of each artery was obtained with its false negative ratio (FNR). Pearson correlation coefficient $(r)$ values were calculated to test the association between Doppler vascular parameters and gestational age. A level of statistical significance ( $P$ value) of $\leq 0.05$ was considered significant. 


\section{Results}

Table 1 shows that mean gestational age (GA) was 36.7 weeks with a range between 26-42 and SD of 3.8, the mean age of the patients was 29 years with a range between 15-45years and SD of 6.1. For Doppler indices, the mean and SD of MCA, Umbilical (Umb.A) and Uterine A (Ut.A) were calculated as shown in Table 1.The mean of MCA, Umbilical A. and Uterine A. $\mathrm{RI}$ were $0.7,0.6$ and 0.6 respectively, and for PI the mean of MCA and Umb. A were 1.4 and 1.1 respectively. To evaluate Doppler ultrasound findings of the uteroplacental and fetoplacental circulations, the sensitivity of each artery and its FNR are shown in Table 2. For the MCA, among 100 risky patients, 53 patients had abnormal reading, so the sensitivity was $53 \%$ and FNR was $47 \%$. For the Umb.A, there were 52 patients with abnormal reading with a sensitivity of $52 \%$ and FNR of $48 \%$. There were 82 patients with abnormal readings of the uterine artery. Accordingly, the sensitivity was $82 \%$ and FNR was $12 \%$. When results were combined for all the three arteries, it appeared that 29 patients had all three arteries affected,42 patients had two arteries affected, and 13 patients had only one artery affected, but among the remaining 16 patients no artery was affected, so the sensitivity was $84 \%$ and FNR was $16 \%$.

Table 1: Maternal age, gestational age and main Doppler ultrasound findings for 100 risky pregnant women.

\begin{tabular}{lcccc}
\hline Factors & Min. & Max. & Mean & SD \\
\hline Age of patients (years) & 15.0 & 45.0 & 29.0 & 6.1 \\
Gestational age (weeks) & 26.0 & 42.0 & 36.7 & 3.8 \\
Middle cerebral artery RI & 0.3 & 0.9 & 0.7 & 0.1 \\
Middle cerebral artery PI & 0.5 & 3.0 & 1.4 & 0.5 \\
Umbilical artery RI & 0.3 & 1.1 & 0.6 & 0.1 \\
Umbilical artery PI & 0.4 & 2.7 & 1.1 & 0.4 \\
Uterine artery RI & 0.3 & 0.8 & 0.6 & 0.1 \\
\hline
\end{tabular}

SD: Standard deviation; RI: Resistive index; PI: Pulsatility index.

Table 2: Doppler U/S evaluation of feto-placental circulation of the patients.

\begin{tabular}{llccc}
\hline & & No. (\%) & Sensitivity\% & FNR\% \\
\hline Middle cerebral artery & Abnormal & $53(53.0)$ & \multirow{2}{*}{$53 \%$} & $47 \%$ \\
& Normal & $47(47.0)$ & & \\
Umbilical artery & Abnormal & $52(52.0)$ & $52 \%$ & $48 \%$ \\
& Normal & $48(48.0)$ & & \\
Uterine artery & Abnormal & $82(82.0)$ & & $18 \%$ \\
& Normal & $18(18.0)$ & $82 \%$ & \\
Any affected artery & Three arteries & $29(29.0)$ & & \\
& Two arteries & $42(42.0)$ & & \\
& One artery & $13(13.0)$ & $84 \%$ & \\
& Normal & $16(22.0)$ & & \\
Total & & $100(100)$ & & \\
\hline FNR: False negative ratio & & & & \\
\hline
\end{tabular}


The correlation between gestational age and different parameters was analyzed according to gestational age as shown in Table 3. Figure 1 displays scatter diagrams for the same correlations. Correlation between GA of the patients and RI of MCA was significant and negative $(r=-0.347$; $P<0.001)$, i.e., there was a decrease in $\mathrm{RI}$ seen with advancing gestational age. The r-value between MCA $\mathrm{PI}$ and GA was -0.380 with $(P<0.001)$, which mean the PI decreased as gestational age progressed. For the umbilical artery $\mathrm{RI}$, the $r$-value was $-0.277(P=0.005)$, which indicates a decrease in umbilical artery seen with advancing gestational age. The PI of umbilical artery showed an r-value of $-0.310(P=0.002)$, which indicates a decrease in PI of the umbilical artery with increasing gestational age. The r-value for uterine artery RI correlation with GA was $0.026(P=0.795)$, which designates no significant change in the RI with advancing gestational age.

Table 3: Correlation of the Doppler ultrasound indices with gestational age (GA) of 100 risky pregnant women.

\begin{tabular}{llllccc}
\hline & & MCA RI & MCA PI & UA RI & UA PI & UtA RI \\
\hline \multirow{2}{*}{ Gestational age in weeks } & $r$ & -0.347 & -0.380 & -0.277 & -0.310 & 0.026 \\
& $P$ & $<0.001$ & $<0.001$ & 0.005 & 0.002 & 0.795 \\
\hline
\end{tabular}

$r=$ Pearson correlation coefficient.
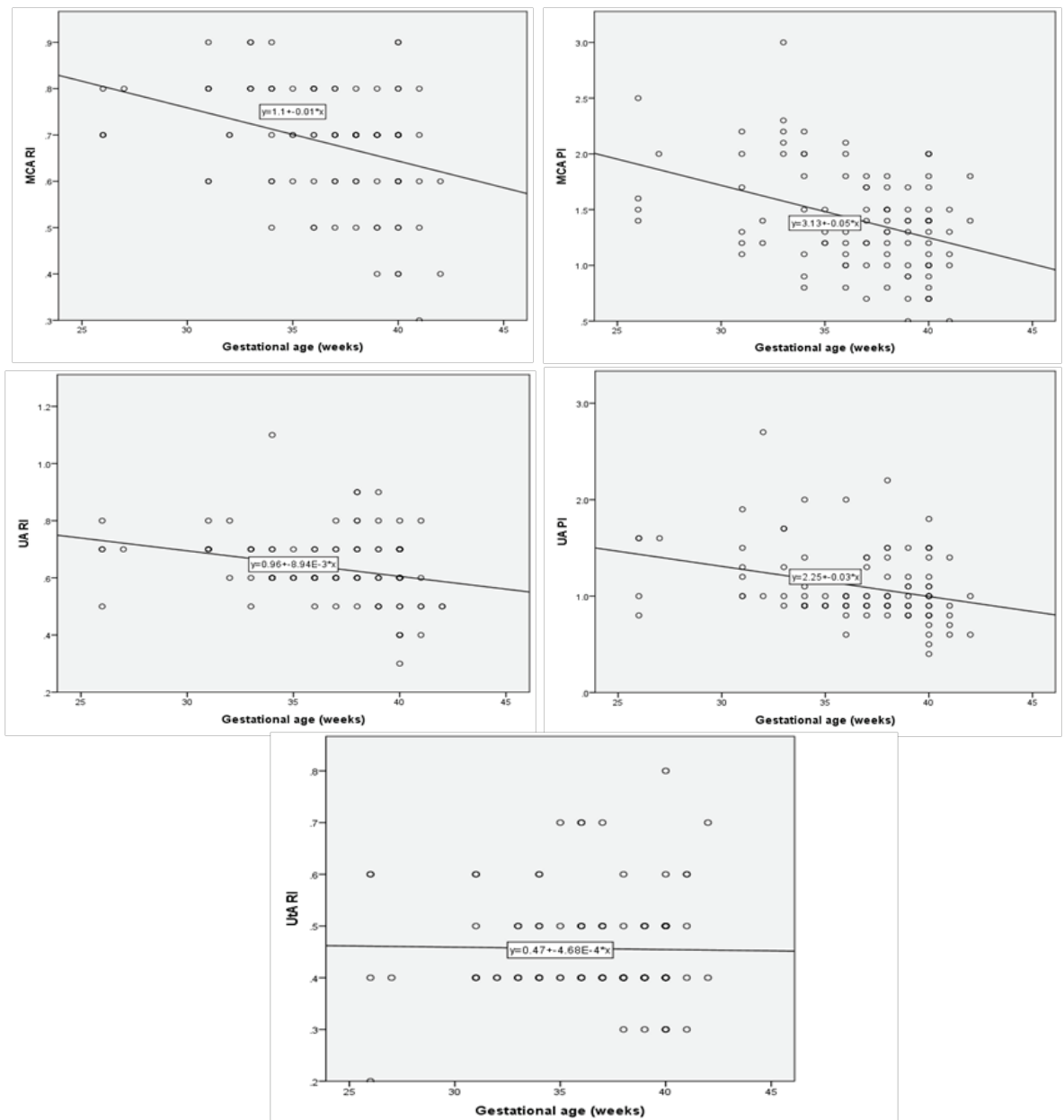

Figure 1: Scatter diagrams of the correlation between various Doppler vascular indices and gestational age in weeks ( $r$-values and their $P$ values are shown in Table 3 ). 
Table 4 shows that the most common Doppler vascular patterns among pregnant women in early third trimester were having abnormal three arteries. In mid third trimester, the commonest pattern was having no or one affected artery. In late third trimester, the predominant pattern having two affected arteries.

\section{Discussion}

Delivery of healthy offspring is the ultimate goal of a breeding program; Doppler ultrasound causes a significant decrease in perinatal mortality and morbidity (Nicholson). ${ }^{14}$ The usefulness of the RI and $\mathrm{PI}$ of Umbilical A, and MCA, as well as RI of maternal Uterine $A$ were evaluated to detect risky fetus among risky pregnancy. For MCA evaluation, the most dependent index was the PI, which had a mean of 1.4, and then RI, which had a mean of 0.7 in the current study. The sensitivity of MCA was $53 \%$, which was very close to a study done by Shnieder ${ }^{15}$ having a sensitivity of $52.8 \%$, while in comparison with another Indian study done by Sachin ${ }^{16}$ the sensitivity of fetal MCA was only $16.7 \%$, but according to their study, normal MCA $\mathrm{PI}$ and RI may not be an indicator of fetal well-being. A prenatal death was observed in one pregnant lady, during her follow up by Doppler which had shown a change in the MCA indices from abnormal to normal. This suggests that normalization of the MCA indices during chronic hypoxia may not be an indicator of fetal well-being as in severe fetal hypoxia, the brain-sparing effect breaks down due to acidemia or brain edema, and the low MCA indices becomes normal, such finding had been noticed in a study done by Sachin ${ }^{16}$ which supports our finding. Therefore, detection of these changes from abnormal to normal MCA indices with prolonged hypoxia, predicts prenatal death. Correlating these indices with the gestational age, these indices were regressing as gestational age was advancing, and in our study, the correlation of MCA with gestational age was statistically significant with a $P$ value of $<0.001$ for both $\mathrm{RI}$ and PI. These findings were similar to Sachin $^{16}$ who found falling of indices after 28weeks of gestation, reflecting a decrease in vascular resistance with increasing gestational age. Similar findings were also noticed in an Iranian study done by Bano. ${ }^{18}$ A study in Germany done by Tarzamni $^{18}$ and a study done in the USA by Baschat. ${ }^{19}$ The correlation coefficient of Umb.A RI was $(r=-0.277, P=0.005)$ and for PI was ( $\mathrm{r}=-0.310, P=0.002)$ which are highly significant. The sensitivity of UA in our study was $52 \%$, which is comparable to the result of an Indian study by Shnieder $;^{15}$ the sensitivity was $57.1 \%$. This relatively higher sensitivity of the UA is probably because it directly reflects the resistance in the placental vascular bed. Comparing the current results with results of Sachin ${ }^{16}$ who

Table 4: The distribution of affected arteries among gestational age categories of pregnant women with IUGR.

\begin{tabular}{lcccc}
\hline $\begin{array}{l}\text { No. of affected } \\
\text { Arteries }\end{array}$ & $\begin{array}{c}\mathbf{2 6 - 3 4} \\
\text { No. (\%) }\end{array}$ & $\begin{array}{c}\text { Gestational age (weeks) } \\
\text { No. (\%) }\end{array}$ & $\begin{array}{c}\mathbf{3 9 - 4 2} \\
\text { No. (\%) }\end{array}$ & $\begin{array}{c}\text { Total } \\
\text { No. }\end{array}$ \\
\hline Nil & $5(22.7)$ & $11(50.0)$ & $6(27.3)$ & 22 \\
One artery & $10(23.3)$ & $19(44.2)$ & $14(32.6)$ & 43 \\
Two arteries & $7(29.2)$ & $2(8.3)$ & $15(62.5)$ & 24 \\
Three arteries & $4(36.4)$ & $3(27.3)$ & $4(36.4)$ & 11 \\
Total no. (\%) & $26(26.0)$ & $35(35.0)$ & $39(39.0)$ & $100(100.0)$ \\
\hline$P$ value (Fisher's exact test) $=0.028$ & & & \\
\hline
\end{tabular}


obtained a sensitivity of $79.2 \%$ of UmbA, the difference in the results could be due to their small sample size (only 40 cases). In the examination of Ut.A the most commonly used indices is the Rl; the mean of $\mathrm{RI}$ among 100 risky pregnant women was 0.6 and this is regarded as abnormal, because Flischer and Bowe ${ }^{7,8}$ found that after 26weeks of gestation, the normal range of Ut.A $\mathrm{RI}$ is between 0.45-0.58, consequently raised $\mathrm{RI}$ above 0.58 is considered abnormal. The sensitivity of Ut.A RI in the present study was $82 \%$, when compare with the study of Arduini, ${ }^{20}$ who found the sensitivity of Ut.A RI $71.4 \%$. The correlation between RI of Ut.A and gestational age was not significant in the current study (0.026 with a $P=0.795)$. This agrees with findings of Antasklis ${ }^{21}$ who found the physiological process of trophoblastic invasion is reflected in the observation from Doppler studies that impedance to flow in the uterine arteries decreases with gestation between 6 and 24 weeks and remains constant thereafter, so no changes occur with advancing gestational age. The most frequently affected number of arteries as revealed by Doppler studies was three abnormal arteries in the gestational age between 26-34 weeks, one abnormal artery between 35-38 weeks, and two abnormal arteries between 39-42 weeks. These patterns probably reflect the varying severity of intrauterine growth restriction throughout the third trimester.

\section{Conclusion}

Doppler ultrasound is important in predicting pregnancy at risk women, helping in their management and aiding in the decision of their deliveries. Umbilical artery and fetal middle cerebral artery Doppler indices are correlated strongly and inversely with gestational age. A weak correlation between maternal Uterine A Doppler indices and gestational age was seen in late pregnancy. Two arteries examination yields more information than one artery.

\section{Conflicts of interest}

The authors report no conflicts of interest.

\section{References}

1. Giles WB, Trudinger BJ, Cook CM. Fetal umbilical artery flow velocity-time waveforms in twin pregnancies. Br J Obstet Gynaecol 1985; 92: 490-7.

2. Giles WB, Trudinger BJ, Baird PJ. Fetal umbilical artery flow velocity waveforms and placental resistance: pathological correlation. $\mathrm{Br} \mathrm{J}$ Obstet Gynaecol 1985; 92:31-8.

3. Arbielle P. Fetal arterial Doppler - IUGR and hypoxia. Eur J Obstet Gynecol 1997; 75:51-3.

4. Adamson SL. Arterial pressure, vascular input impedance, and resistance as determinants of pulsatile blood flow in the umbilical artery. Eur $\mathrm{J}$ Obstet Gynecol 1999; 84:119-25.

5. Arabin B, Bergmann PL, Saling E. Simultaneous assessment of blood flow velocity waveforms in uteroplacental vessels, the umbilical artery, the fetal aorta and the fetal common carotid artery. Fetal Ther 1987; 2:17-26.

6. Ertan A. Doppler Sonography in obstetrics. Donald school text book of ultrasound in obstetrics and gynecology. 2nded. London New York Washington Baco Raton: Parthenon Publishing group; 2008. p. 521-33.

7. Fleischer A, Schulman H, Farmakides G, Bracero L, Blattner P, Randolph G. Umbilical artery flow velocity waveforms and intrauterine growth retardation. Am J Obstet Gynecol 1985; 151:5025.

8. Bowe S, Vyas S, Campbell S, Color Doppler imaging of uterine artery in pregnancy; normal ranges of impedance to blood flow, mean velocity and volume flow. Ultrasound Obstetric Gynecol 1992; 2.61-256:

9. Allan $P$, McDicken N, Pozniak M, Dubbins $P$. Clinical doppler ultrasound. 2nded. Curchill Livingstone: Elsevier; 2006. p. 14-5.

10. Brosens I, Dixon HG, Robertson W. Fetal growth retardation and the arteries of the placental bed. Br J Obstet Gynaecol 1977; 84:656-64.

11. Trudinger BJ, Cook CM, Giles WB, Fong E, Connelly A, Wilcox W. Fetal umbilical artery velocity waveform and subsequent neonatal out com. Br J Obestet Gynecol 1991;98: 378-84.

12. Fleischer A, Toy E, Lee W, Manning F, Romero R. Sonography in obstetric and Gyncology. 7th ed. McGraw Hill Professional; 2011. p. 258.

13. Tekay A, Campbell S. Ultrasonography in Obstetrics and Gynaecology. 4th ed. Philadelphia: Saunders; 2011. P. 677-717.

14. Nicholson S, Nimrod .Doppler Assessment of Pregnancy. Diagnostic Ultrasound. 1st ed. St. Louis: Mosby; 1991. p. 955-68.

15. Schneider KT, Loos W. The 10th anniversary of obstetric Doppler sonography-development and perspectives. Geburtshilfe Frauenheilkd 1989; 49:407-15. 
16. Sachin Kh, Umesh $P$, Shazia B, Samarjit B, Anurag B. Comparison of Diagnostic efficacy of umbilical artery and middle cerebral artery waveform with color Doppler study for Detection of intrauterine growth retardation. Journal of Obstetrics and Gynecology of India 2013; 64 (4):249-55.

17. Bano S, Chaudhary V, Pande S, Mehta V, Sharma A. Color Doppler evaluation of cerebral-umbilical pulsatility ratio and its usefulness in the diagnosis of intrauterine growth retardation and prediction of adverse perinatal outcome.Indian J Radiol Imaging 2010; 20(1):205.

18. Tarzamni KM, Nezami N, Sobhani N, Eshraghi N, Tarzamni $\mathrm{M}$ and Talebi $\mathrm{Y}$. Nomograms of Iranian fetal middle cerebral artery Doppler wave form and uniformity of their pattern with other population nomograms. BMC Pregnancy Childbirth 2009; 8(1):50.

19 . Baschat A, Gembruch U. The cerebroplacental Doppler ratio revisited. Ultrasound Obstet Gynecol 2003; 21:124-7.

20. Arduini D, Rizzo G. Normal values of Pulsatility Index from fetal vessels: a cross sectional study on 1556 healthy fetuses. J Perinat Med 1990; 18:65-72.

21. Antasklis A, Doskalakis G, Tzortzis E, Mechalas S. The effect of gestational age and placental location in the prediction of pre-eclampsia by uterine artery Doppler velocity in low risk nuliparous women. Ultrasound Obestet Gynecol 2000; 16:635-9.

22. Plasencia W, Maiz N, Poon L, Yu C, Nicolaides $\mathrm{KH}$. Uterine artery Doppler in the prediction of pre-eclampsia. Ultrasound Obstet Gynecol 2008; 32(2):138-46. 